\title{
The GEYSERS Concept and Major Outcomes
}

\author{
Anna Tzanakaki ${ }^{1}$, Sergi Figuerola ${ }^{2}$, Joan A. García-Espín ${ }^{2}$, \\ Dimitra Simeonidou ${ }^{3}$, Nicola Ciulli ${ }^{4}$, Philip Robinson ${ }^{5}$, Juan Rodríguez ${ }^{6}$, \\ Giada Landi ${ }^{4}$, Bartosz Belter ${ }^{7}$, Pascale Vicat-Blanc ${ }^{8}$, Matteo Biancani ${ }^{9}$, \\ Cees de Laat ${ }^{10}$, Eduard Escalona ${ }^{2}$, and Artur Binczewski ${ }^{7}$ \\ ${ }^{1}$ Athens Institute of Technology \\ atza@ait.gr \\ ${ }^{2}$ i2CAT Foundation \\ ${ }^{3}$ University of Bristol \\ ${ }^{4}$ Nextworks \\ ${ }^{5}$ SAP Ireland \\ ${ }^{6}$ Telefónica I+D \\ ${ }^{7}$ Poznan Supercomputing and Networking Center \\ ${ }^{8}$ Lyatiss \\ ${ }^{9}$ Interoute Italy \\ ${ }^{10}$ University of Amsterdam
}

\section{Introduction}

Large-scale computer networks supporting both communication and computation are extensively employed to deal with a variety of existing and emerging demanding applications. These high-performance applications, requiring very high network capacities and specific IT resources, cannot be delivered by the current Best Effort Internet. Optical networking is offering a very high capacity transport with increased dynamicity and flexibility through recent technology advancements including dynamic control planes etc. The European project GEYSERS (Generalised Architecture for Dynamic Infrastructure Services) proposed a novel architecture capable of provisioning "Optical Network and IT resources" for end-to-end service delivery. The proposed approach adopts the Infrastructure as a Service (IaaS) paradigm. The GEYSERS architecture presents an innovative solution to enable infrastructure operators to virtualize their optical network + IT physical resources and offer them as a service based on the user/application requirements. The adoption of Virtual Infrastructures (VIs) facilitates sharing of physical resources among various virtual operators, introducing new business models that suit well the nature and characteristics of the Future Internet and enables new exploitation opportunities for the underlying Physical Infrastructures (PIs).

The GEYSERS architecture (figure 1) is based on a layered model that introduces two novel layers, the enhanced Network Control Plane (NCP+) and the Logical Infrastructure Composition Layer (LICL), and leverages on existing solutions to represent a Service Middleware Layer (SML) and the PI. The PI layer comprises optical network + IT resources from different providers. These resources are virtualized by the LICL and composed into VIs using dynamic on-demand planning mechanisms. On top of LICL, GEYSERS deploys the NCP+, a control plane based on the Generalised 


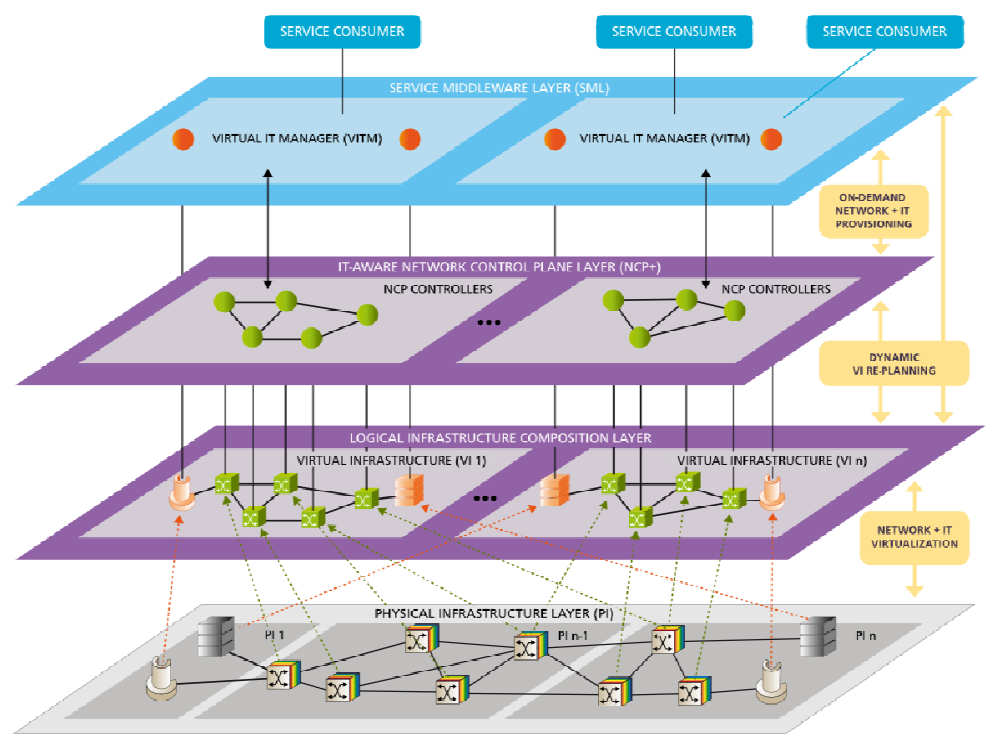

Fig. 1. GEYSERS layered architecture

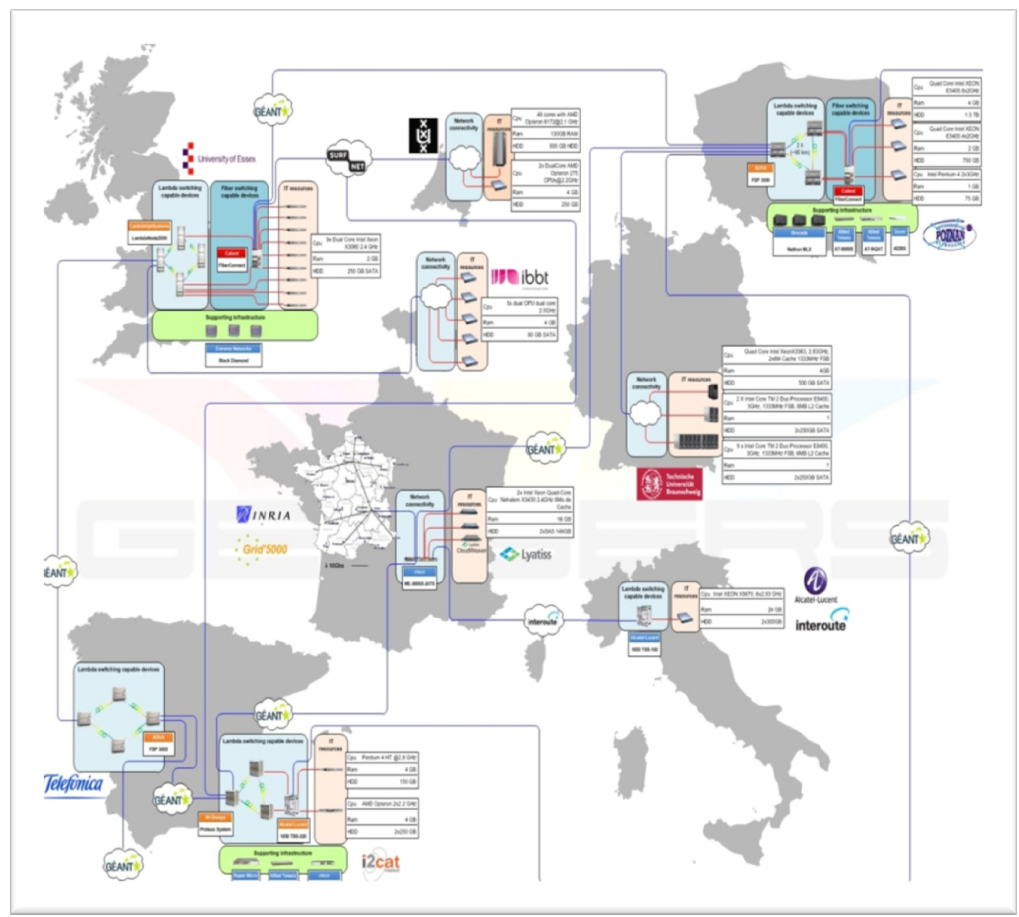

Fig. 2. GEYSERS testbed 
Multi-protocol Label Switching (GMPLS) and the Path Computation Element (PCE) that operates over each VI and controls the virtual resources. The $\mathrm{NCP}+$ is responsible for the path computation and the allocation of both network and IT resources. Finally, SML is responsible for translating application requests and service level agreements (SLAs) into technology specific parameters to trigger the service provisioning procedures of GEYSERS.

\section{Project Outcomes}

During the project lifetime the details of the proposed architecture have been specified and a novel framework for joint optical network and IT infrastructure automated planning, provisioning and operation have been developed. Through this the physical resources are dynamically partitioned and composed in VIs and are offered to operators as a Service. GEYSERS provides a multi-vendor and multi-domain solution, supporting interoperability with legacy technologies, decoupling of control and physical layers together with the required coherence mechanisms between them as well as energy-efficient, scalable and trustable joint consideration of NET + IT resources.

LICL includes the software architecture that abstracts, virtualises and composes optical Network and IT infrastructure resources, and enables dynamic infrastructure planning and re-planning. The existing implementation facilitates custom, scheduled, on-demand profiling of the VIs and exposes virtual resource control and management interfaces to different roles involved in the GEYSERS ecosystem (PI provider, VI provider \& VI operator). In addition, it empowers a novel mechanism to allow telecom operators to decouple the infrastructure from the service offering. LICL addresses security, information modelling, access control, SLA management and synchronization issues between VIs and PIs. The support LICL provides to the networking and IT resources (extensible to other resources), is based on NDL and VXDL data models and parameterizes physical and virtual resources with all the required attributes and was demonstrated at FuNeMS 2012 and the Telefonica I+D Dissemination event.

The NCP+ operating over the virtual optical network infrastructures acts as an enabler for the integration of dynamic optical network services in cloud computing. It dynamically cooperates with the Service Middleware Layer and offers on-demand provisioning of network transport services, coupled with IT resources connected to the edges. $\mathrm{NCP}+$ supports new connection paradigms including assisted unicast, restricted anycast and full anycast. In addition, it offers scheduled/advance reservations and cross-layer escalation of monitoring and recovery procedures for global service resilience. Deployment and experimental validation of the $\mathrm{NCP}+$ has been performed at the Uninversity of Bristol and the PSNC testbeds, while Public demonstrations of unicast \& anycast services have also been performed at FuNeMS 2012 and the Future Networks 10th FP7 concertation meeting.

The GEYSERS Optical Test-beds provide an integration, validation and demonstration platform (figure 2) and comprise five core optical nodes (WDM/DWDM, OTN and fibre switces) interconnected with high speed links over GÉANT, GLIF and 
CBF infrastructures and IT Nodes, offering computing clusters and other IT facilities, attached to the core nodes through 1 GE link. The IT resources (servers, PC clusters and Storage elements) are provided through eight cloud nodes. Examples of experiments performed using the test-bed include: application of virtualization paradigms to optical infrastructure resources and validation of seamless and efficient infrastructure composition from IT and network resources, experiments with ondemand provisioning of enhanced network connectivity services tailored to specific applications requirements over a VI.

To facilitate GEYSERS outcomes to complement existing products and services and be able to be integrated with existing systems, GEYSERS has focused on playing an active role in standardization bodies such as the Internet Engineering Task Force (IETF), the TeleManagement Forum (TMF), the Distributed Management Task Force (DMTF), the Open Grid Forum (OGF), the National Institute of Standards and Technologies (NIST) and the VXDL forum.

Open Access. This article is distributed under the terms of the Creative Commons Attribution Noncommercial License which permits any noncommercial use, distribution, and reproduction in any medium, provided the original author(s) and source are credited. 